\title{
Atenuación de huella de adquisición guiada por atributos sísmicos
}

\author{
Acquisition Footprint Attenuation Driven by Seismic Attributes
}

\author{
Cuellar-Urbano Mayra \\ Facultad de Ingeniería \\ Departamento de Ingeniería Geofísica \\ Universidad Nacional Autónoma de México \\ Correo:mayra_q23@yahoo.com.mx
}

\author{
Chávez-Pérez Sergio \\ Dirección de Exploración y Producción \\ Instituto Mexicano del Petróleo \\ Correo:sergio.chavezp@gmail.com
}

Información del artículo: recibido: septiembre de 2012, aceptado: abril de 2013

\begin{abstract}
Resumen
Parte de los problemas en la representación sísmica que PEMEX afronta, es la presencia de la huella de adquisición en los datos, manifestado como ruido altamente correlacionable con la geometría de adquisición y presente en todos los datos sísmicos adquiridos, tanto en tierra como en mar. Este ruido dificulta la interpretación de rasgos estratigráficos y estructurales en rebanadas de tiempo, generando artefactos numéricos en el cálculo de atributos sísmicos útiles para la interpretación sísmica. La huella de adquisición permanece a pesar de las medidas tomadas durante la adquisición y el procesamiento sísmico para que sea mínima. Se utilizó un flujo de trabajo de atenuación guiado por atributos sísmicos geométricos con los que se realza la huella de adquisición para obtener un modelo de huella sintética que se resta adaptablemente de datos sísmicos. Para ilustrarlo se utilizaron datos proporcionados por PEMEX Exploración y Producción.
\end{abstract}

\footnotetext{
Abstract

Acquisition footprint, one of the major problems that PEMEX faces in seismic imaging, is noise highly correlated to the geometric array of sources and receivers used for onshore and offshore seismic acquisitions. It prevails in spite of measures taken during acquisition and data processing. This pattern, throughout the image, is easily confused with geological features and misguides seismic attribute computation. In this work, we use seismic data from PEMEX Exploración y Producción to show the conditioning process for removing random and coherent noise using linear filters. Geometric attributes used in a workflow were computed for obtaining an acquisition footprint noise model and adaptively subtract it from the seismic data.
}

\section{Descriptores:}

- geofísica

- sismología de exploración

- representación sísmica

- huella de adquisición

- atributos sísmicos

\section{Keywords:}

- geophysics

- exploration seismology

- seismic imaging

- acquisition footprint

- seismic attributes 


\section{Introducción}

Actualmente la adquisición sísmica 3D es un método de prospección geofísica importante en la exploración de hidrocarburos en PEMEX Exploración y Producción, en la planeación de localizaciones de perforación y en la extracción de crudo. Sin embargo, durante la adquisición y el procesamiento se genera ruido incluso después de tomar las medidas necesarias para minimizarlo.

La huella de adquisición es ruido presente en todos los datos sísmicos adquiridos tanto en tierra como en mar debido a la disposición de fuentes y receptores. La geometría de adquisición queda impresa en los datos generando falsos rasgos geológicos como karsticidad y enmascarando otros como fracturas, lo que dificulta la correcta interpretación sísmica en horizontes sísmicos y en rebanadas de tiempo, al igual que genera falsos rasgos geológicos en el cálculo de atributos sísmicos. El análisis de atributos sísmicos proporciona información detallada de las propiedades de esa información sísmica.

Se utilizaron herramientas de mejoramiento de imágenes con las que se suprimieron también otros tipos de ruido para mejorar la representación sísmica y evitar que se generen artefactos numéricos que alteran el resultado de cálculos posteriores de posprocesamiento. Se realizó el cálculo de los atributos sísmicos geométricos de coherencia o similitud y curvatura para guiar la atenuación y con el fin de mejorar los resultados de su cómputo. En este trabajo seguimos una metodología para eliminar el ruido relacionado con la huella de adquisición en una etapa de posprocesamiento de datos.

\section{Huella de adquisición}

La huella de adquisición es cualquier patrón de ruido altamente correlacionable con la distribución geométrica de fuentes y receptores en la superficie terrestre. Este patrón repetitivo se genera debido a la periodicidad de las distancias fuente al receptor (offset) dentro de cada celda ( $\mathrm{bin}$ ) formada por las líneas de receptores y fuentes (Falconer y Marfurt, 2008). Así, la huella es resultado de las decisiones tomadas antes, durante y después de la adquisición. Es necesario identificar el patrón de la huella de adquisición para poder atenuarla.

El diseño de adquisición define la distribución particular de celdas y de sus propiedades (figura 1), como apilamiento, azimut y distancia fuente a receptor (Canning y Gardner, 1998), parámetros que se suponen constantes, pero que en realidad es difícil que lo sean debido a las obstrucciones y limitaciones en campo. Estos parámetros están definidos por los intervalos $E$ en- tre fuentes y $\boldsymbol{e}$ entre receptores. De estos últimos depende la huella de adquisición (Meunier, 2011) tomando en cuenta que en adquisiciones 2D las coordenadas son 3, el tiempo $(t)$ y dos coordenadas espaciales: puntos de tiro $\left(x_{s}\right)$ y receptores $\left(x_{r}\right)$. En las adquisiciones 3D son 5 antes de apilar, las ya mencionadas y dos más, coordenadas de tiros $\left(y_{s}\right)$ y coordenadas de receptores $\left(y_{r}\right)$ (Vermeer, 2009).

La huella también es causada por arribos de ondas convertidas, múltiples, ruido generado por fuentes de adquisición, modelos inadecuados de velocidad de procesamiento, correcciones estáticas deficientes, ruido coherente y patrones irregulares de apilamiento, entre otros (Hill et al., 1999). Además, el ruido aleatorio contribuye a variaciones laterales de amplitud traza a traza entre los conjuntos apilados por punto medio común (CMP, Common Midpoint). La huella es más fuerte en secciones someras, donde hay menor apilamiento y huecos de información debido a submuestreos (Falconer y Marfurt, 2008), es visible aún a mayor profundidad (Cvetkovic et al., 2008).

Utilizamos los datos de un volumen sísmico proporcionado por PEMEX Exploración y Producción para ejemplificar este proceso de atenuación. En la figura 2 mostramos la rebanada de tiempo en $0.5 \mathrm{~s}$, con amplitudes originales después del procesamiento convencional de datos sísmicos. El carácter de los rasgos relacionados con la huella cambia conforme avanza en tiempo, dado que este patrón es visible aún en las secciones transversales afectando la continuidad de los reflectores u ocultando rasgos geológicos.

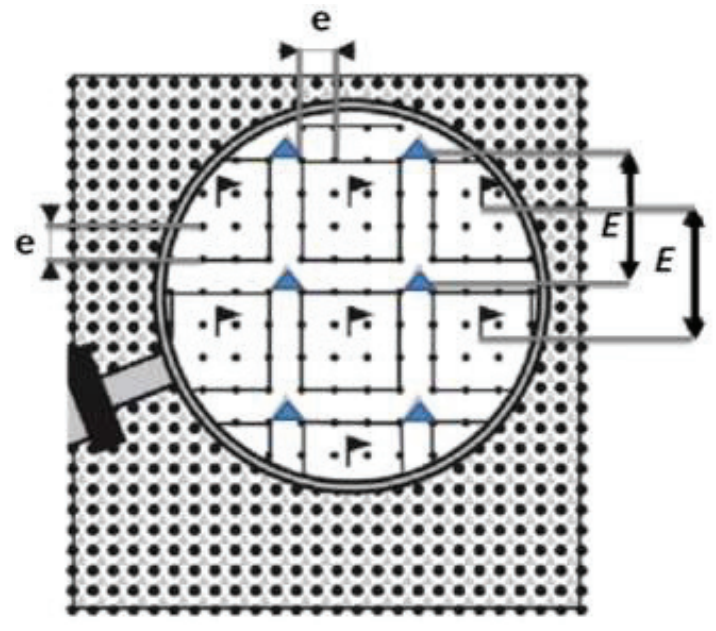

Figura 1. Diseño de adquisición óptimo (Meunier, 2011) con disposición de fuentes y receptores con distancia fuente a receptor $\boldsymbol{E}$. El intervalo entre geófonos es $\mathbf{e}$ y las banderas señalan cada grupo de fuentes y receptores 


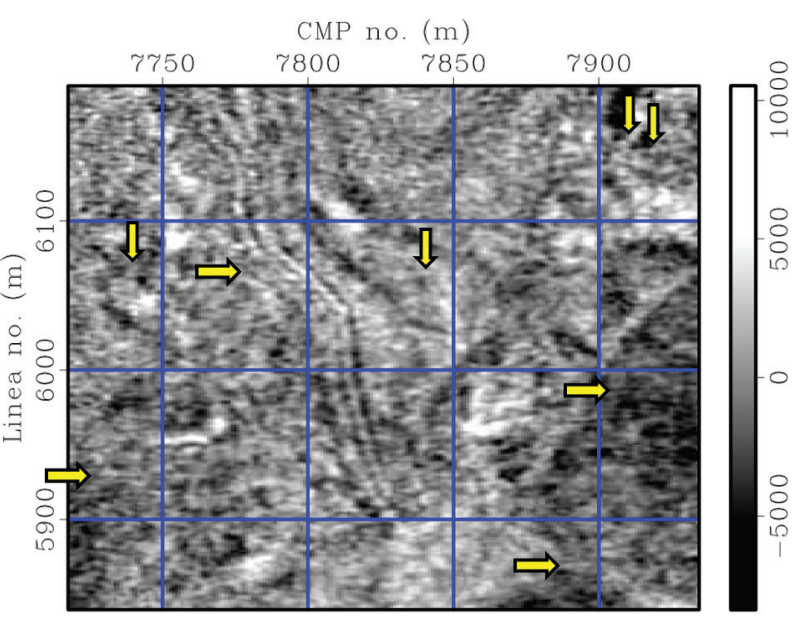

Figura 2. Rebanada de tiempo en $0.5 \mathrm{~s}$ de amplitudes originales del volumen sísmico del área Vigilante ubicado en el Activo Integral Burgos. Las flechas indican el efecto de la geometría de adquisición en la representación sísmica

En principio, una forma de obtener una adquisición que resulte en la mejor representación sísmica del subsuelo, tanto en las amplitudes sísmicas originales como en los atributos sísmicos, es elegir o diseñar una geometría de adquisición correcta. Este diseño debe contemplar el muestreo correcto de los datos, de modo que al menos dos de cuatro de las coordenadas espaciales puedan estar representadas de manera apropiada. El buen muestreo de estas coordenadas y el uso de aperturas más amplias entre estaciones evita el empalme de la señal (Schuster y Liu, 2001), generando una mejor reconstrucción del campo de ondas continuo del subsuelo y minimizando el efecto de la huella de adquisición.

a)

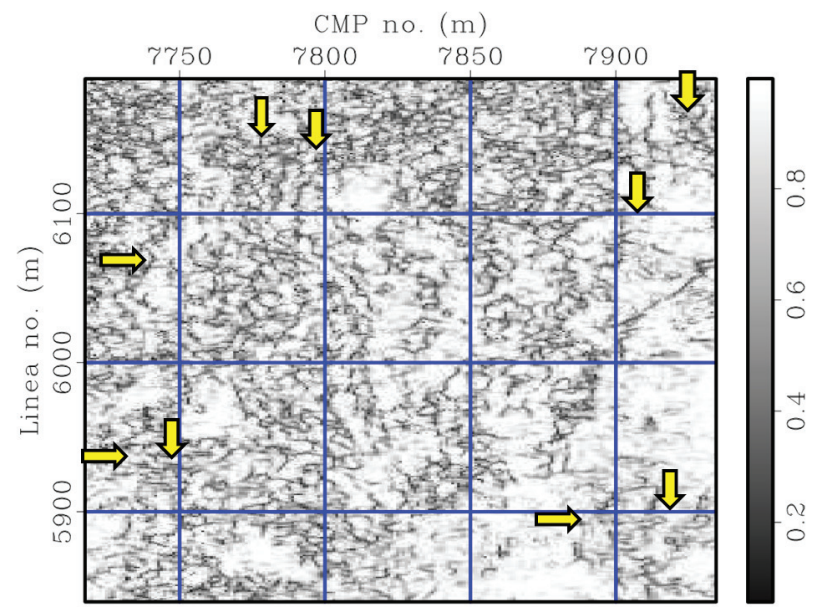

Posteriormente, para el cálculo de atributos, la huella de adquisición genera artefactos numéricos en la representación, lo que ocasiona obtener cálculos erróneos que entorpecen la interpretación. Prueba de ello se puede observar en la figura 3, donde se muestra el resultado del cálculo de los atributos de coherencia y curvatura de amplitud más positiva en la rebanada de tiempo en $0.5 \mathrm{~s}$.

\section{Atenuación de huella de adquisición}

El flujo de trabajo que se describe a continuación utiliza atributos geométricos y filtros que buscan adaptarse a las estructuras con el fin de preservarlas, pero que en este caso sirven como una herramienta para conservar y realzar la huella de adquisición. Los atributos proporcionan una forma de resaltar ruido relacionado con la huella de adquisición para identificarlo y sustraerlo adaptablemente del volumen de amplitudes sísmicas (Falconer y Marfurt, 2008).

Existen diversas técnicas utilizadas para atenuar la huella de adquisición en una etapa de posprocesamiento, entre ellas, la atenuación guiada por atributos sísmicos geométricos ha probado ser efectiva para eliminar ruido mientras preserva mejor las estructuras geológicas. Los atributos sísmicos geométricos, como coherencia y curvatura, permiten la predicción de fracturas y de rasgos estratigráficos del subsuelo debido a que son sensibles a cambios laterales de amplitud. En este caso, tales atributos permiten realzar los componentes de la huella de adquisición debido a su periodicidad espacial.

b)

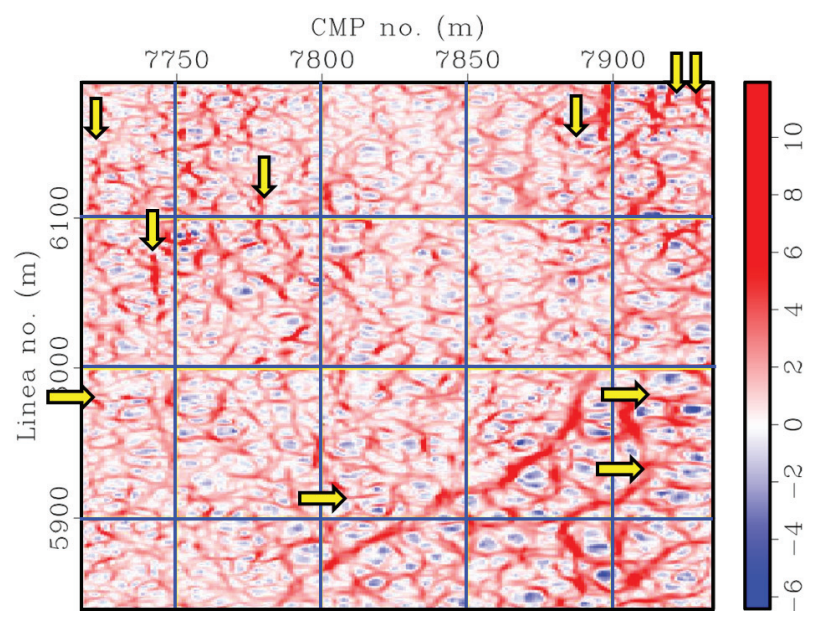

Figura 3. Atributo de a) coherencia y b) curvatura de amplitud para la rebanada de tiempo en $0.5 \mathrm{~s}$, donde es posible ver la contaminación de la información sísmica debido a la huella de adquisición. Las flechas indican dónde es más visible este componente de ruido, tanto en dirección longitudinal como transversal 
Existen varios métodos que se utilizan para realzar el contenido en frecuencias en los datos sísmicos, con el fin de obtener atributos sísmicos con mejor resolución lateral y vertical, libres de artefactos que contaminen la imagen para obtener una mejor estimación de la huella de adquisición. Entre esas técnicas se encuentran el filtrado de componentes principales, el filtrado orientado a estructuras y sus variantes, el filtrado $f-k$ y filtrado $k_{x^{-}}$ $k_{y^{\prime}}$ entre otros (tabla 1$)$.

Tabla 1. Técnicas utilizadas en la eliminación de huella de adquisición; adaptado de (Cvetkovic et al., 2008).

\begin{tabular}{ll}
\hline & FTT 2D $\mathrm{k}_{\mathrm{x}}-\mathrm{k}_{\mathrm{y}}$ \\
& Transformada de ondícula discreta 2D y \\
& transformada de ondícula estacionaria 2D \\
& $\begin{array}{l}\text { Inversión acústica y anisótropa (VTI) de } \\
\text { forma de onda completa (Gholami et al., 2011) }\end{array}$ \\
\hline 3D & FKK o $\mathrm{k}_{\mathrm{x}}-\mathrm{k}_{\mathrm{y}}-\omega$ \\
& Transformada de Radon \\
& Transformada de ondícula 3D y transformada \\
& de curvículas (curvelets) 3D \\
Otras & Filtros combinados (Al-Dossary y Marfurt, \\
herramientas & 2007) \\
& Transformada de ondícula compleja 1D \\
& Filtro de echado local vs filtro de echado \\
& regional \\
& Migración de cuadrados mínimos \\
& Rotación de los datos sísmicos a los ejes de \\
& muestreo (Ng y Zheng, 2011) \\
& Inversión de datos sísmicos guiados por la \\
& información de la geometría de adquisición \\
& (Zabihi et al., 2012) \\
\hline
\end{tabular}

El flujo de procesamiento mostrado está basado en varias de estas técnicas que se adaptan para lograr una mejor preservación de información sísmica útil. El flujo de trabajo, por lo tanto, no es único y depende tanto de la información sísmica como del criterio del geocientífico que lleve a cabo el procesamiento.

\section{Atenuación guiada por atributos}

Falconer y Marfurt (2008) realizaron el planteamiento del flujo de trabajo para atenuar huella de adquisición guiado por atributos sísmicos que ayuda a enfatizar la huella y que hacen factible su caracterización. En la figura 4 podemos observar que para llevar a cabo el flujo de trabajo se necesitan dos volúmenes de entrada, el volumen de datos sísmicos y el volumen de un atributo sísmico geométrico calculado a partir de los datos originales.

El flujo de atenuación consiste en localizar la información relacionada con ruido específico de huella de adquisición para sustraer estas amplitudes al volumen original de datos. El acondicionamiento de datos debe cumplir con generar una respuesta donde se haya realzado ruido de adquisición en los atributos sísmicos geométricos.

El ruido coherente de baja velocidad también pertenece al rango de frecuencias bajas, por lo que es útil primero filtrar las amplitudes sísmicas con un pasabajas para excluir la señal de alta frecuencia no contaminada (Marfurt et al., 2011).

\section{Cálculo de atributos sísmicos geométricos}

Se utilizaron dos tipos de atributos sísmicos geométricos (Chopra y Marfurt, 2010; Marfurt et al., 1998) que realzan la huella de adquisición.

- Coherencia. Es la medida de similitud existente entre dos o más trazas. Este atributo es una herramienta que ayuda a detectar y realzar discontinuidades estructurales o estratigráficas y cambios abruptos difíciles de identificar en la señal sísmica (Chopra y Marfurt, 2008). El cálculo de coherencia se realiza a través de un análisis de ventanas en conjuntos de trazas, entre las que se establece estadísticamente el valor de mayor similitud (varianza) y, a través del cual, se realiza el análisis discreto del echado y límites de las estructuras geológicas (Marfurt et al., 1998; Bahorich y Farmer, 1995).

- Curvatura. Es la propiedad bidimensional de una curva que describe qué tan arqueado (cóncava o convexa) es un punto específico. Se define como la razón de cambio de dirección de una curva y es el recíproco del radio de un círculo tangente a una curva en un punto donde su valor es mayor a medida que la curva difiere de una línea recta, e igual a cero si es esta última y mide los cambios laterales $\mathrm{y}$ verticales sutiles en el echado, que usualmente son opacados por deformaciones regionales mayores (Chopra y Marfurt, 2007; Bahorich y Farmer, 1995).

Una vez calculados los atributos con huella de adquisición realzada, utilizando ventanas del tamaño de las celdas y filtros suavizadores orientados a estructuras que realzan el contenido de alta frecuencia (Marfurt, 2006), el objetivo principal del flujo es estimar el componente de ruido relacionado con la geometría de adquisición (una huella sintética del estudio) presente en los datos sísmicos. Para este volumen, elegimos el atributo de coherencia (Marfurt et al., 1998) que mejor resaltó la huella (figura 5) y un filtro lineal de media orientado a estructuras (Al-Dossary y Wang, 2011) para 

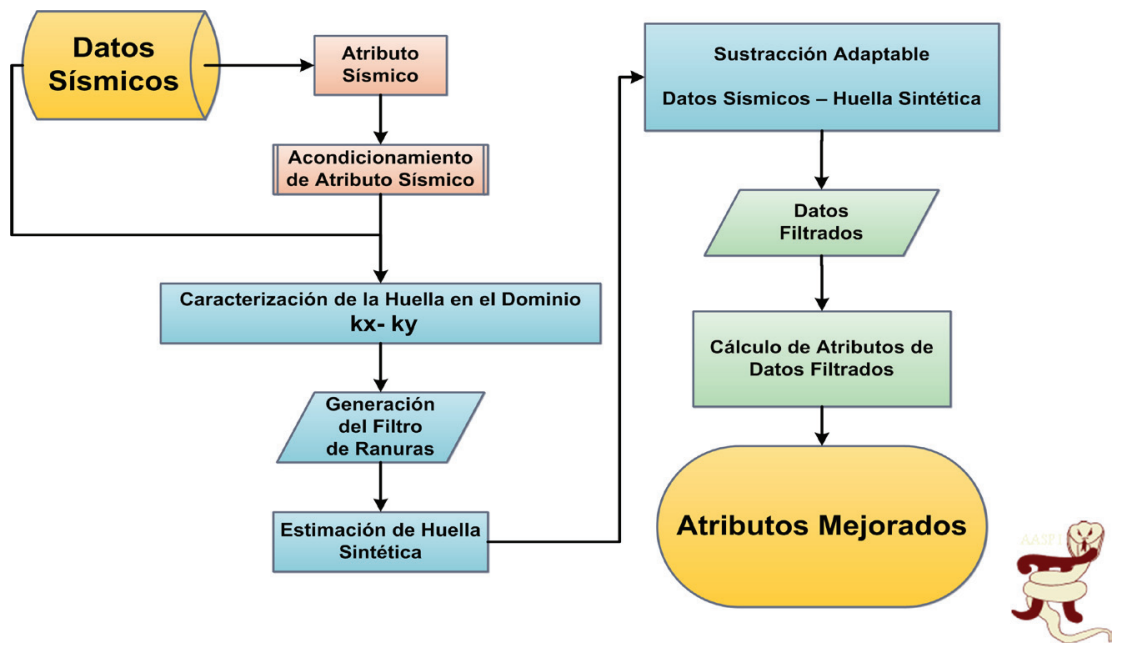

Figura 4. Flujo de atenuación de huella de adquisición; adaptado de (Marfurt et al., 2011)

atenuar picos de amplitud e información no relacionada con los componentes de ruido de la huella.

\section{Caracterización de la huella de adquisición en domino $k_{x}-k_{y}$ (dominio de Fourier)}

Por la periodicidad del diseño de la adquisición se generan artefactos periódicos en los componentes de fase y amplitud de los datos que, al transformar al dominio de Fourier, son posibles de identificar y suprimir. Distintos errores de adquisición y procesamiento dan lugar a cambios en la forma de onda, amplitud y echado. También existen artefactos generados durante la migración, al corregir por efecto del echado del reflector, por el empalme del operador, así como errores sistemáticos en el análisis de velocidad. Los atributos son sensibles a componentes de longitudes de onda relativamente corta de la huella de adquisición y hacen de los atributos una excelente herramienta en la caracterización de la huella.

El volumen de datos sísmicos y el volumen del atributo acondicionado se transforman en rebanadas de tiempo para su procesamiento (Marfurt et al., 2011). En esta metodología utilizamos filtros $k_{x}-k_{y}$ usualmente utilizados en la eliminación de ruido estructurado, tanto periódico como no periódico, en la construcción de imágenes sísmicas (figura 6). La información de baja frecuencia centrada en el origen está relacionada con la información sísmica útil y la de alta frecuencia detectable está relacionada con datos anómalos no relacionados con la geología.

En este dominio establecimos un umbral de circunferencia $k_{x}^{2}+k_{y}^{2}=0.03$ con centro en el origen de ambos ejes $k_{x}=0$ y $k_{y}=0$ para delimitar la zona que se preservará intacta durante la atenuación de la huella de adquisi- ción (figura 6). La huella de adquisición corresponde a los componentes periódicos de alta amplitud en el espectro. Entonces, en el espectro $k_{x}-k_{x^{\prime}}$ la cercanía al origen de ambos ejes indica longitudes de onda mayores y mientras se aleja, se refiere a longitudes de onda más cortas. Los eventos perfectamente planos estarán referidos a $k_{x}-k_{y}=0$. Por lo tanto, la mayoría de los rasgos geológicos corresponden a la superficie $k_{x}^{2}+k_{y}^{2}<k_{\max }^{2}$, donde $k$ es el valor del radio del umbral, con centro en el origen del mapa $k_{x}-k_{y}$.

Esta elección del umbral se realiza comparativamente entre los espectros del atributo y de los datos sísmicos originales para elegir el área que es más importante conservar. Una mala elección del umbral repercute en procesos posteriores de procesamiento eliminan-

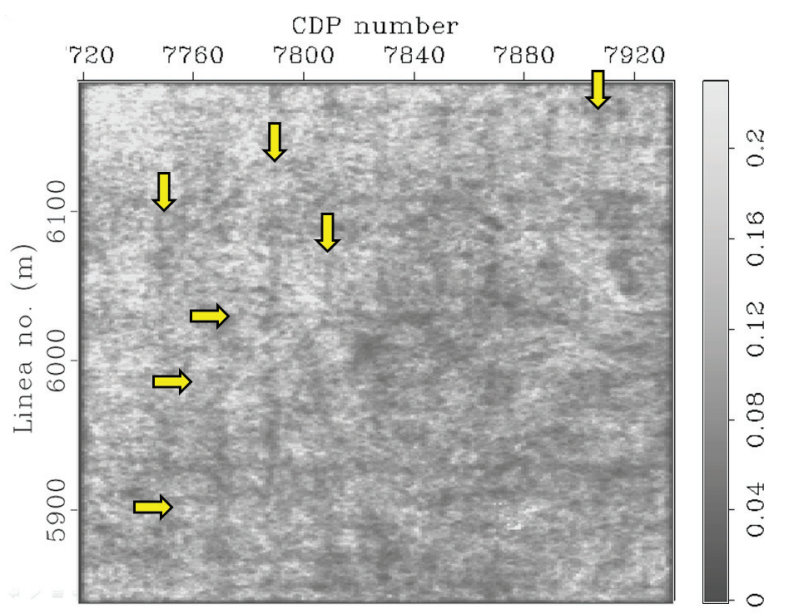

Figura 5. Atributo de coherencia suavizado por un filtro de media controlado por un factor de $25 \%$. Las flechas señalan rasgos relacionados con huella de adquisición. Estos rasgos son identificables debido a que son líneas casi paralelas en dirección longitudinal y transversal 
a)

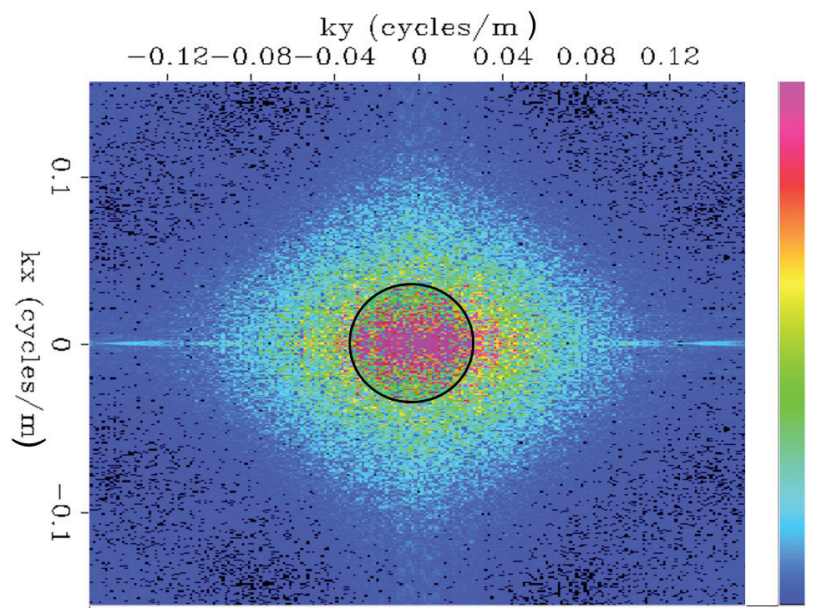

b)

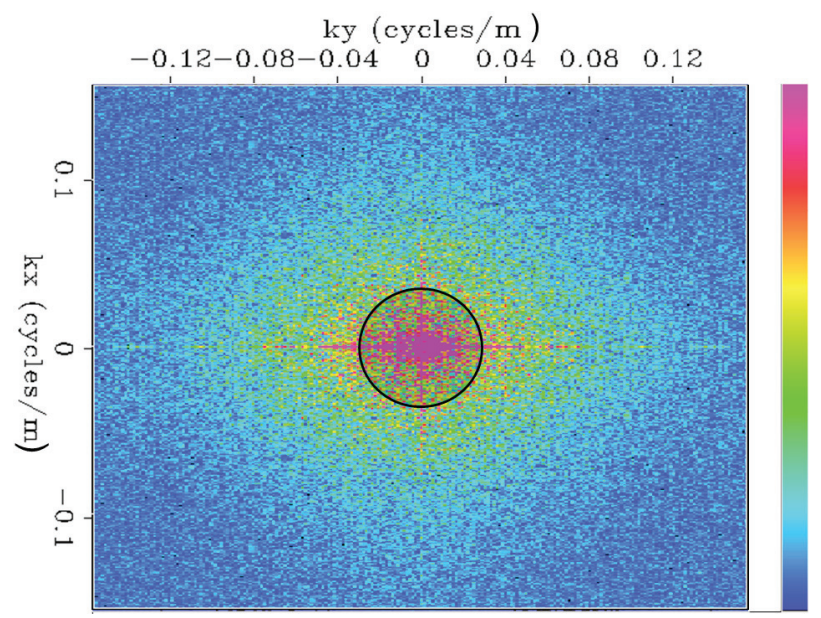

Figura 6. Componentes espectrales de a) las amplitudes sísmicas originales y b) del atributo sísmico al transformar al dominio $k_{\mathrm{x}}-k_{\mathrm{y}}$. La circunferencia con centro en el origen establece un umbral bajo el cual actuará el filtro de ranuras, con un radio $k=0.03$ ciclos $/ \mathrm{m}$

do componentes de información sísmica útil. De la elección del umbral dependerá la estimación de la huella de adquisición y el éxito del flujo de atenuación.

\section{Filtro de ranuras}

Una vez determinado el umbral que establece qué componentes se mantendrán intactos, se estiman los componentes anómalos relacionados con la huella de adquisición para la elaboración de una máscara o filtro de ranuras que permita el paso de los componentes $k_{x}-k_{y}$ de alta amplitud asociados con la huella, excluyendo la zona $k_{x}^{2}+k_{y}^{2}<k_{\max }^{2}$ asociada con rasgos geológicos y estructurales (figura 7).

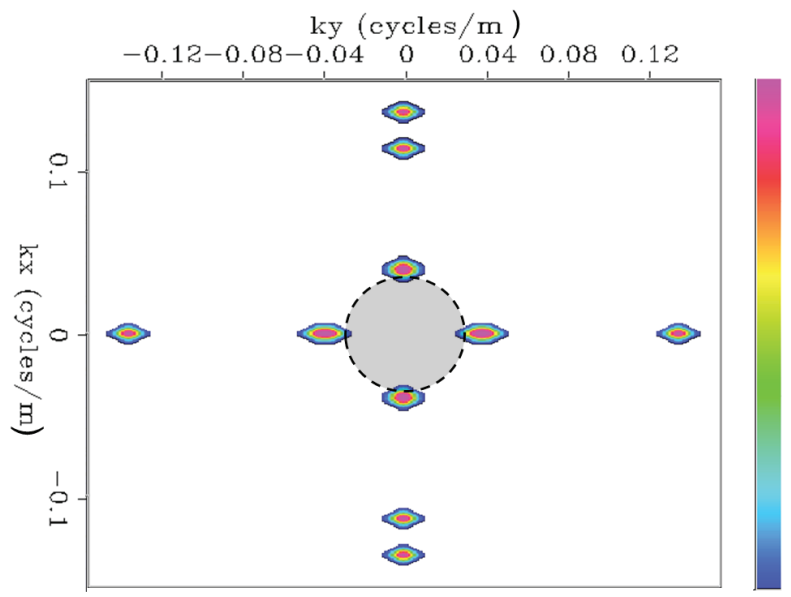

Figura 7. Filtro de ranuras que permite pasar los componentes de alta amplitud relacionados con la huella de adquisición en la rebanada de tiempo en $0.5 \mathrm{~s}$. Cada filtro de ranura resaltado sobre los ejes está referido a algún componente de la huella de adquisición
El resultado de aplicar este filtro de ranuras al volumen de amplitudes sísmicas originales, se regresa al dominio cartesiano, resultando en una huella de adquisición estimada (figura 8). Este resultado también es útil para establecer un control de calidad sobre la elección del radio del umbral en el dominio $k_{x}-k_{y}$ y permite determinar si el filtro actuó sobre el ruido o si afectó la señal.

Para cada rebanada de tiempo el filtro que se genera es diferente, ya que la huella de adquisición es diferente para cada tiempo. Lo que obtenemos es una versión sintética de la huella impresa en la adquisición y, en este caso, del volumen sísmico Vigilante (figura 8) es posible observar que este filtro resalta la periodicidad de los componentes de la huella de adquisición, tanto vertical como horizontalmente. En la adquisición, es usual que la geometría no sea perfecta y por ello algunos componentes serán diferentes para cada zona del volumen.

\section{Sustracción adaptable}

Una vez obtenida la huella sintética, se remueve la huella del volumen de datos sísmicos (Falconer y Marfurt, 2008) con aproximaciones de cuadrados mínimos en una sustracción adaptable (Marfurt et al, 2011). En una situación ideal se esperaría que esta huella se pudiera sustraer de la información sísmica directamente. Sin embargo, existen cambios laterales en la geometría de adquisición debido a los inconvenientes de adquisición ya descritos. Esta técnica se utiliza para la eliminación de múltiples de periodo largo y para minimizar el error entre los datos originales $\left(d_{i j}\right)$ y la versión estimada del ruido $\left(\eta_{i j}\right)$ 


$$
\min \varepsilon^{2}=\sum_{k=1}^{K}\left(\sum_{j=-M_{y}}^{M_{y}} \sum_{i=-M_{x}}^{M_{x}} d_{l(k)+i, m(k)+j}-\alpha_{k} \omega_{i, j} \eta_{l(k)+i, m(k)+j}\right),
$$

donde

$\varepsilon$ es el error,

$d_{l(k)+i, m(k)+j}$ son los datos dentro de la $k$-ésima versión ventaneada de los datos originales,

$\omega_{i, j}(x, y)$ es la función 2D que genera los factores para un mejor ajuste del ruido a los datos originales,

$\alpha_{k}$ es la amplitud del $k$-ésimo punto de control y

$\eta_{l(k)+i, m(k)+j}$ es la $k$-ésima versión ventaneada del ruido estimado.

Reduciendo la ecuación anterior obtenemos la siguiente expresión en forma matricial

$$
\alpha_{k}=\left[w n^{T} w n+\beta I\right]^{-1} w n^{T} d,
$$

donde $\beta$ es un factor de preblanqueo e $I$ es la matriz identidad.

Estos parámetros previenen la división entre cero cuando existen trazas muertas. Los valores generados por esta ecuación crean el mejor ajuste de la función de ruido con los datos sísmicos (Falconer y Marfurt, 2008).

Podemos observar el resultado de llevar a cabo la atenuación asistida por atributos con la sustracción adaptable entre el volumen de amplitudes sísmicas y la

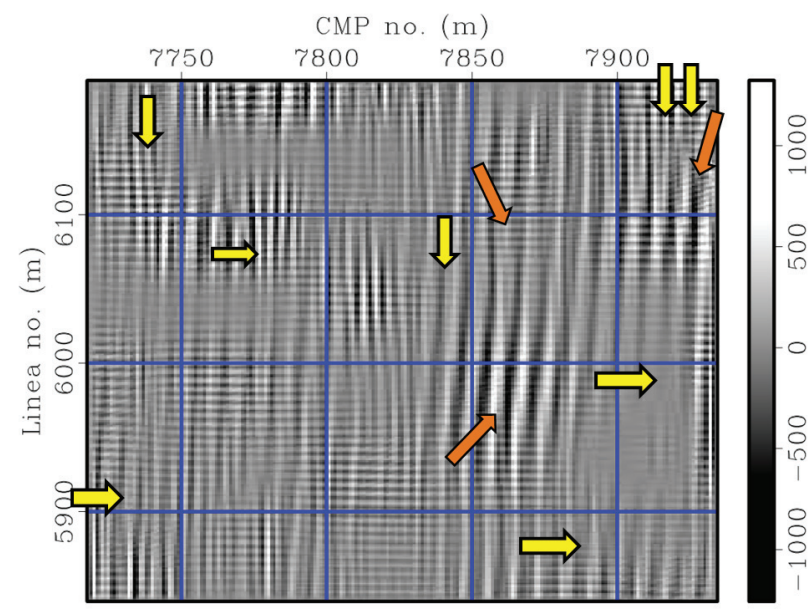

Figura 8. Versión sintética de la huella de adquisición de la rebanada de tiempo en $0.5 \mathrm{~s}$ con una escala de colores maximizada para apreciar los rasgos de ruido realzado. Las flechas señalan los distintos tipos de ruido realzados, donde predomina la huella de adquisición. Las flechas amarillas (en la impresión, claras) señalan rasgos verticales y horizontales, las flechas naranja (oscuras) señalan rasgos relacionados con otros tipos de ruido huella estimada a partir del filtro de ranuras generado del atributo sísmico geométrico en la figura 9.

Ciertos rasgos regionales fueron resaltados debido a la sustracción del ruido, lo cual mejora la relación señal a ruido de los datos. La finalidad de llevar a cabo esta metodología es obtener atributos sísmicos donde sean más fáciles de identificar los cambios estructurales en la representación sísmica y se refleja principalmente en el cálculo de atributos sísmicos geométricos, útiles en la interpretación estructural de los datos sísmicos adquiridos en una zona de interés petrolero.

\section{Resultados del cálculo de atributos mejorados}

Realizamos el cálculo de huella de adquisición guiando el flujo de trabajo con el atributo de coherencia (figura 10) de modo que al volver a calcular este atributo y el de curvatura (figura 11), disminuyera el impacto del ruido aún presente en los datos sísmicos. Se observa que, en comparación con la figura 4a, para el atributo de coherencia se pueden delimitar mejor algunas discontinuidades señaladas por las flechas amarillas donde indicamos variaciones laterales de amplitud predominantes en la representación sísmica para esta rebanada de tiempo en la figura 10.

Las flechas centrales (color rosa, oscuro en la impresión) señalan discontinuidades que son más fáciles de observar, las flechas amarillas (claro) señalan donde habían artefactos por huella de adquisición que fueron atenuados, y las flechas verdes (gris medio) señalan rasgos lineales en dirección de la huella longitudinal y transversal, que no pertenecen a ruido y están relacionados con las estructuras geológicas.

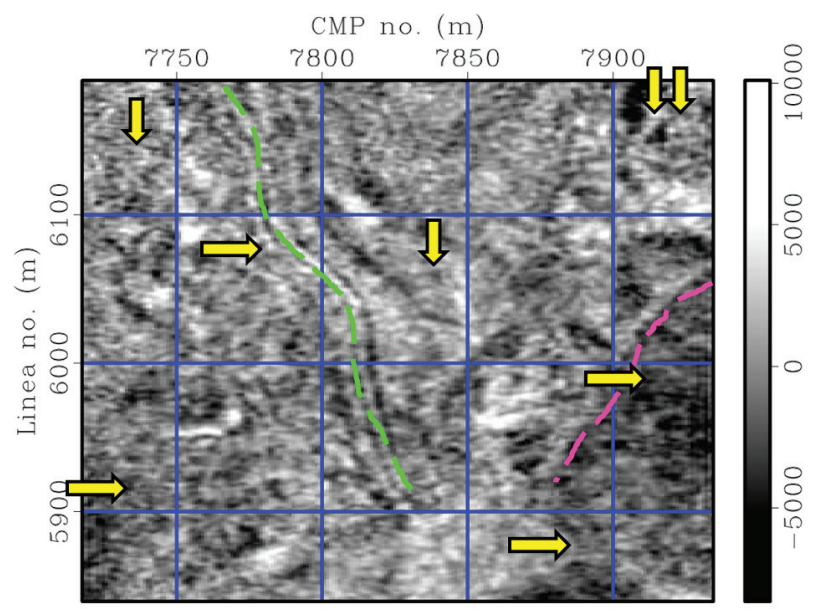

Figura 9. Rebanada de tiempo en $0.5 \mathrm{~s}$ filtrada por el flujo de atenuación guiado por el atributo de coherencia. Las líneas punteadas realzan rasgos geológicos que se conservaron y mejoraron 


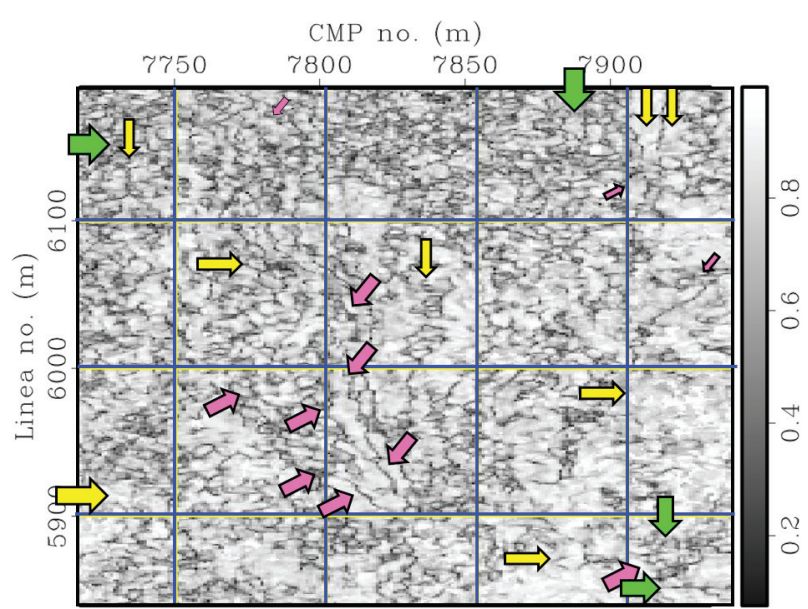

Figura 10. Atributo de coherencia calculado después de filtrar el volumen de Vigilante por el flujo de atenuación de huella de adquisición guiado por el atributo de coherencia. Rebanada de tiempo en $0.5 \mathrm{~s}$

Para el atributo de curvatura (figura 11), comparando con el resultado mostrado del cálculo de curvatura de amplitud, previo a efectuar la atenuación de huella de adquisición (figura $4 \mathrm{~b}$ ), es posible notar que los rasgos señalados con flechas en esa imagen, ya no son representativos en esta otra, y ahora es posible identificar más rasgos estructurales (flechas amarillas o claras).

Con el resultado de curvatura de amplitud, hay rasgos relacionados con estructuras difíciles de detectar en el cálculo previo a la atenuación de la huella. En esta representación de la rebanada en $0.5 \mathrm{~s}$ podemos observar rasgos que también son visibles en el atributo de coherencia donde esas estructuras se delinean y señalamos con flechas negras rasgos relacionados con estas estructuras. Se indica con flechas amarillas (claras) las áreas donde había afectación por huella de adquisición. Las flechas azules (oscuras), a su vez señalan un rasgo importante y fácilmente reconocible a lo largo de los resultados de cada uno de los pasos de procesamiento que, sin embargo, pierde relevancia al observar el atributo de curvatura de amplitud, calculado con los resultados de filtrar o atenuar la huella con el atributo de coherencia y con curvatura de amplitud.

\section{Conclusiones}

La huella de adquisición depende de los datos sísmicos adquiridos. El resultado obtenido del flujo de trabajo para caracterizar a la huella de adquisición será diferente con cada atributo utilizado y mantendrá su expresión característica de líneas paralelas. Aunado a ello,

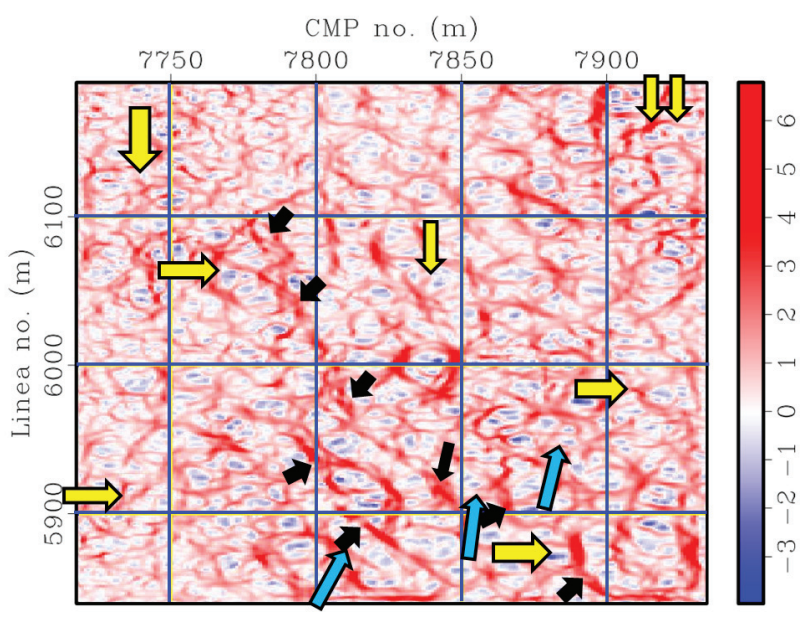

Figura 11. Atributo de curvatura de amplitud calculado después de filtrar el volumen de Vigilante por el flujo de atenuación de huella de adquisición guiado por el atributo de coherencia. Rebanada de tiempo en $0.5 \mathrm{~s}$

este ruido muestra también cierta periodicidad generada por procesos de otras etapas del tratamiento de los datos y todos estos rasgos se llegan a confundir con estructuras geológicas o litológicas que repercuten en mala interpretación y elección de horizontes para la elección de zonas de producción.

Al aplicar esta metodología, el ruido de adquisición se detectó y atenuó. Cada resultado, con los diferentes atributos, resalta ciertas características de la señal sísmica que permiten localizar y delimitar estructuras relevantes para llevar a cabo la interpretación estructural de la zona de exploración. Por ello, debe ser un proceso iterativo en el que el intérprete tiene que ser capaz de identificar la huella de adquisición desde un principio para conservar estás estructuras mientras se atenúa la huella de adquisición.

Existe una gran mejora en el resultado con el cálculo de atributos sísmicos geométricos en la metodología, ya que permite detectar mejor las estructuras del subsuelo, enmascaradas por huella de adquisición. Este flujo de trabajo es una opción para acelerar el proceso de posprocesamiento de datos utilizando equipo computacional no especializado, variando la parametrización para obtener resultados con imágenes mejoradas.

Para el personal de PEMEX Exploración y Producción tiene un gran impacto utilizar la metodología en los proyectos de exploración y explotación de yacimientos. La eficiencia y rapidez del resultado permite al intérprete cerciorarse de los resultados al realizar correlaciones más exactas con otros tipos de información geofísica y geológica obteniendo una mejor representación del subsuelo. 


\section{Agradecimientos}

A Marco Vázquez García, gerente de estudios geofísicos de PEMEX Exploración y Producción, por permitir el uso de datos sísmicos y la publicación. A Rodolfo Rocha-Ruiz, de PEMEX Exploración y Producción, por su apoyo y por facilitar el acceso a los datos sísmicos de Vigilante. Al Instituto Mexicano del Petróleo y a la Gerencia de Estudios Geofísicos de PEMEX Exploración y Producción por el financiamiento para la realización de este trabajo.

\section{Referencias}

Al-Dossary S., Marfurt K.J. Lineament-Preserving Filtering. Geophysics, volumen 72 (número 1), enero-febrero de 2007: 1-8.

Al-Dossary S., Wang Y.E. Structure-Preserving Smoothing for 3D Seismic Attributes. SEG Expanded Abstracts, volumen 30, 2011: 1004-1008.

Bahorich M. y Farmer S. 3-D Seismic Discontinuity for Faults and Stratigraphic Features. The Coherence Cube. The Leading Edge, volumen 14, octubre de 1995: 1053-1058.

Canning A. y Gardner G.H.F. Reducing 3-D Acquisition Footprint for 3-D DMO and 3-D Prestack Migration. Geophysics, volumen 63 (número 4), julio-agosto de 1998: 1177-1183.

Chopra S. y Marfurt K.J. Volumetric Curvature Attributes for Fault/Fracture Characterization. First Break, volumen 25, julio de 2007: 19-30.

Chopra S. y Marfurt K.J. Integration of Coherence and Volumetric Curvature Images. The Leading Edge, volumen 29, septiembre de 2010: 1092-1107.

Chopra S. y Marfurt K.J. Emerging and Future Trends in Seismic Attributes. The Leading Edge, volumen 27, marzo de 2008: 298-318.

Cvetkovic M., Pralica N., Falconer S., Marfurt K.J., Chávez-Pérez S. Comparison of Some Algorithms for Acquisition Footprint Suppression and Their Effect on Attribute Analysis. SEG Expanded Abstracts, volumen 27, 2008: 2637-2641.
Falconer S. y Marfurt K.J. Attribute-Driven Footprint Suppression. SEG Expanded Abstracts, volumen 27, 2008: 26672671.

Gholami Y., Brossier R., Operto S., Ribodetti A., Virieux, J., Acoustic VTI Full Waveform Inversión: Sensitivity Analysis and Realistic Synthetic Examples. SEG Expanded Abstracts, volumen 30, 2001: 2465-2470.

Hill S., Schultz M., Brewer S. Acquisition Footprint and Fold-ofStack Plots. The Leading Edge, volumen 18 (número 6), junio de 1999: 686-695.

Marfurt K.J., Davogustto O., Dowdell B., Guo S., Kwiatkowski J.T., Roy A., Verm S., Zhang B. Section 14 Attribute Assisted Footprint Suppression Workflow, Running AASPI Software with GUIs (manual de usuario), 1a ed., EEUU, Universidad de Oklahoma, 2011, pp. 1-13.

Marfurt K.J. Robust Estimates of 3D Reflector Dip and Azimuth. Geophysics, volumen 71, julio-agosto de 2006: P29-P40.

Marfurt K.J., Lynn-Kirlin R., Farmer S.L., Bahorich M.S. 3-D Seismic Attributes Using a Semblance-Based Coherency Algorithm. Geophysics, volumen 63 (número 4), julio-agosto de 1998: 1150-1165.

Meunier J. Seismic Acquisition from Yesterday to Tomorrow, 1a ed., EEUU, Society of Exploration Geophysicists, 2011, pp. 85, 135-136.

Ng M., Zheng Y. Multi-Orientation Footprint Attenuation Using Coordinate Rotations on 3D Data. SEG Expanded Abstracts, volumen 30, 2011: 3678-3682.

Schuster G.T., Liu Z. Seismic Array Theorem and Rapid Calculation of Acquisition Footprint Noise. Geophysics, volumen 66 (número 6), noviembre-diciembre de 2001: 1843-1849.

Vermeer-Gijs J.O. 3D Seismic Survey Design, 1a ed., EEUU, Society of Exploration Geophysicists, 2009, pp. 17-19.

Zabihi-Naeini E., Hoeber H., Campbell S. A New Approach to Reducing Multiple Leakage on Time Lapse Datasets, en: EAGE Conference \& Exhibition incorporating SPE EUROPEC 74 , 2012, Copenhagen. Responsibly Securing our Natural Resources, EAGE Copenhagen'12, 2012, p. 5. 


\section{Este artículo se cita:}

\section{Citación estilo Chicago}

Cuellar-Urbano, Mayra, Sergio Chávez-Pérez. Atenuación de huella de adquisición guiada por atributos sísmicos. Ingeniería Investigación y Tecnología, XV, 02 (2014): 199-208.

\section{Citación estilo ISO 690}

Cuellar-Urbano M., Chávez-Pérez S. Atenuación de huella de adquisición guiada por atributos sísmicos. Ingeniería Investigación y Tecnología, volumen XV (número 2), abril-junio 2014: 199-208.

\section{Semblanza de los autores}

Cuellar Urbano-Mayra. Obtuvo el título de ingeniera geofísica en la Facultad de Ingeniería de la UNAM en 2012. Laboró en el Instituto Mexicano del Petróleo en 2011 y hasta junio del 2012. Actualmente labora en la Facultad de Ingeniería, UNAM.

Sergio Chávez-Pérez. Obtuvo los títulos de ingeniero geofísico en 1984 por la Facultad de Ingeniería de la UNAM, el de maestro en ciencias (geofísica) en 1987 por la Universidad de Carolina del Sur, EUA y el de doctor en filosofía (geofísica) en 1997 por la Universidad de Nevada, Reno, EEUU. Actualmente es investigador en el Instituto Mexicano del Petróleo y profesor de asignatura de la Facultad de Ingeniería de la UNAM. Sus áreas de interés profesional son: representación sísmica (migración, modelado, tomografía e inversión), procesamiento de datos sísmicos y transformación de campos de ondas, interpretación de datos sísmicos y propagación de ondas sísmicas. 